\title{
Advancing Pediatric Care in India Requires Further Study of Adverse Childhood Experiences
}

\author{
Susan Kim ${ }^{1} \cdot$ Arifa Zaidi $^{1} \cdot$ Timothy Rice ${ }^{1}$ \\ Received: 7 October 2020 / Accepted: 25 November 2020 / Published online: 8 January 2021 \\ (C) Dr. K C Chaudhuri Foundation 2021
}

To the Editor: Adverse childhood experiences (ACEs), including childhood abuse and neglect, increase the risk for and severity of chronic medical conditions. A history of ACEs increases the risk for development of numerous chronic medical conditions, such as ischemic heart disease, cancer, bronchitis, stroke and diabetes, in addition to psychiatric disorders [1]. To provide optimal health care, we must consider ACEs.

One fifth of the world's child population resides in India. Certain ACEs remain high in India, including abandonment, childhood labor, and street-begging, while healthcare resources directed towards ACEs remain comparatively low [2]. An increase in attention to ACEs may yield long-term gains in pediatric and adult health. The creation and study in India of culturally tailored preventative and therapeutic interventions to address ACEs may additionally provide a model for developing countries.

Evidence-based measures of ACEs may guide further efforts. The original validated ACE questionnaire [1] is a simple 10-item self-administered tool that assesses the number of ACEs experienced during childhood. A novel modified version of the ACEs questionnaire, named the Pediatric ACEs and Related Life-Event Screener (PEARLS), incorporates additional criteria to assess for risk factors shown to affect child and adult health, such as food and housing insecurity, caregiver impairment or death, and community discrimination and violence [3]. These additional criteria may have expanded relevance for children in developing countries.

The paucity of data surrounding the prevalence of ACEs in other countries and the ramifications of cultural variances in response to ACEs highlight an area that require further investigation [4]. Identifying ACEs through improved screening is a first step. Provider-initiated indicated interventions can serve as a nidus for the creation and study of programs to improve lifelong population health.

\section{Compliance with Ethical Standards}

Conflict of Interest None.

\section{References}

1. Felitti VJ, Anda RF, Nordenberg D, et al. Relationship of childhood abuse and household dysfunction to many of the leading causes of death in adults. The adverse childhood experiences (ACE) study. Am J Prev Med. 1998;14:245-58. Available at: http://www.ncbi.nlm. nih.gov/pubmed/9635069.

2. Singhi P, Saini AG, Malhi P. Child maltreatment in India. Paediatr Int Child Health. 2013;33:292-300. Available at: http://www. tandfonline.com/doi/full/10.1179/2046905513Y.0000000099.

3. Koita K, Long D, Hessler D, et al. Development and implementation of a pediatric adverse childhood experiences (ACEs) and other determinants of health questionnaire in the pediatric medical home: a pilot study. PLoS One. 2018;13:e208088 Available at: https://dx. plos.org/10.1371/journal.pone.0208088.

4. Oral R, Ramirez M, Coohey C, Nakada S, Walz A, Kuntz A, Benoit J, Peek-Asa C. Adverse childhood experiences and trauma informed care: the future of health care. Pediatr Res. 2016;79(1-2):227-33. https://doi.org/10.1038/pr.2015.197.

Publisher's Note Springer Nature remains neutral with regard to jurisdictional claims in published maps and institutional affiliations.

Timothy Rice

Timothy.Rice@mountsinai.org

1 Department of Psychiatry, Icahn School of Medicine at Mount Sinai, New York, NY, USA 